\title{
Sedentary behaviour is associated with increased long-term cardiovascular risk in patients with rheumatoid arthritis independently of moderate-to-vigorous physical activity
}

Sally A. M. Fenton ${ }^{1,3^{*}}$, Jet J. C. S. Veldhuijzen van Zanten ${ }^{1,3}$, George D. Kitas ${ }^{1,3}$, Joan L. Duda ${ }^{1}$, Peter C. Rouse ${ }^{4}$, Chen-an $\mathrm{Yu}^{1,5}$ and George S. Metsios, ${ }^{1,2,3}$

\begin{abstract}
Background: Rheumatoid Arthritis (RA) is associated with an increased risk of cardiovascular disease (CVD). The physical dysfunction symptomatic of RA means people living with this disease spend large periods of the day sedentary, which may further elevate their risk of CVD. The primary aim of this study was to investigate relationships between objectively assessed sedentary behaviour patterns and light physical activity (LPA) with 10-year risk of CVD. Secondary aims were to explore the role of sedentary behaviour patterns and LPA for individual CVD risk factors and functional disability in RA. The extent to which associations were independent of moderate-to-vigorous physical activity (MVPA) engagement was also examined.
\end{abstract}

Methods: Baseline data from a subsample of participants recruited to the Physical Activity in Rheumatoid Arthritis (PARA) study were used to answer current research questions. Sixty-one patients with RA (mean age $( \pm S D)=54.92 \pm 12.39$ years) provided a fasted blood sample and underwent physical assessments to evaluate factors associated with their cardiovascular health. Sedentary behaviour patterns (sedentary time, sedentary bouts, sedentary breaks), LPA and MVPA were measured via 7-days of accelerometry. Ten-year CVD risk was computed (Q-risk-score2), and functional disability determined via questionnaire.

Results: Regressions revealed significant positive associations between sedentary time and the number of sedentary bouts per day $\geq 20$ min with 10-year CVD risk, with the reverse true for LPA participation. Associations were independent of MVPA engagement.

Conclusions: Promoting LPA participation and restricting sedentary bouts to $<20$ min may attenuate long-term CVD risk in RA, independent of MVPA engagement.

Trial registration: ISRCTN04121489 (retrospectively registered 19/10/2012).

Keywords: Sedentary behaviour, Physical activity, Rheumatoid Arthritis, Accelerometry, Cardiovascular risk, Inflammation, Physical Function

\footnotetext{
* Correspondence: s.a.m.fenton@bham.ac.uk

'School of Sport, Exercise and Rehabilitation Sciences, University of

Birmingham, Birmingham, UK

${ }^{3}$ Department of Rheumatology, Russells Hall Hospital, Dudley Group NHS

Foundation Trust, Dudley, UK

Full list of author information is available at the end of the article
} 


\section{Background}

Rheumatoid Arthritis (RA) is a chronic, autoimmune inflammatory disease affecting $0.5-1 \%$ of the population, manifesting with joint swelling, cartilage destruction and bone erosion. These clinical characteristics contribute towards joint pain and impaired physical function symptomatic of the disease [1-3]. The heightened inflammatory-load seen in RA may also contribute to the increased risk of cardiovascular disease (CVD) and associated morbidity and mortality [4-10].

It is well evidenced that higher levels of physical activity (PA) associate with improvement in cardiovascular health, systemic inflammation and physical function in RA [11-20]. This relationship is reported to occur in a 'dose-response' manner; with moderate-to-vigorous intensity physical activity [MVPA, i.e., $\geq 3$ metabolic equivalents (METS)] demonstrated to be particularly beneficial for RA outcomes [16-20]. However, recent studies conducted among older adults and non-RA clinical populations, have underlined the role of engagement in light intensity physical activity (LPA, i.e., $1.6-2.9$ METS) for reducing cardiovascular risk, improving metabolic health and attenuating functional disability [21-28]. Thus, engagement in LPA may also be relevant to improving pertinent RA outcomes.

Still, despite evidence highlighting the positive consequences of PA engagement for RA outcomes, people living with RA remain physically inactive, and are often referred to as "sedentary" [29-31]. However, it is important to recognise that whilst both sedentary behaviour and physical inactivity represent the 'non-exercise' part of the physical activity continuum, they are distinct constructs, and can be operationalised as such [11, 32, 33]. Specifically, physically inactivity refers to failure to meet the recommended 150 min of MVPA per week [33, 34], where sedentary behaviour is defined as any waking behaviour resulting in energy expenditure $\leq 1.5$ METS whilst sitting or lying [32, 35].

Sedentary behaviour is demonstrated to be adversely linked to several health outcomes which are relevant to RA. For example, studies have revealed time spent sedentary to hold deleterious consequences for cardiovascular and cardio-metabolic health, as well as functional ability for both healthy adults and patient groups [21, 36-40]. Moreover, the way sedentary time is accumulated is reported to hold implications for CVD risk and physical function. Specifically, the number and length of uninterrupted sedentary periods (i.e., sedentary bouts), and the frequency of interruptions in sedentary time with standing and/or LPA (i.e., sedentary breaks) are reported to be associated with markers of cardiometabolic and cardiovascular health, and physical dysfunction in older cohorts [36, 37, 41-48]. However, research is yet to examine the implications of objectively measured sedentary behaviour patterns and LPA for risk of CVD (and other relevant health outcomes) in RA [49].

The primary aim of this study was therefore to examine the relationships between objectively assessed sedentary time, sedentary behaviour patterns (bouts and breaks) and LPA with global long-term (10-year) CVD risk among people living with RA. The extent to which associations are independent of levels of MVPA participation will also be examined. Indeed, interventions which focus on reducing sedentary behaviour and increasing LPA (i.e., promoting the "sit less, move more" message), will likely only be efficacious towards reducing risk of CVD in the instance these behaviours are favourably associated with CVD risk/profile, independently of the possible cardio-protective effects of MVPA engagement. We hypothesise that higher levels of daily sedentary time, and more frequent (and longer) uninterrupted sedentary bouts, will be adversely associated with 10-year CVD risk, after considering daily levels of MVPA participation. In contrast, we expect that the number of sedentary breaks per day, and higher levels of engagement in LPA, will be beneficially associated with 10-year CVD risk, independently of MVPA engagement. Finally, given the lack of research examining the role of sedentary behaviour patterns and LPA for RA outcomes, secondary aims of this study were to explore the role of sedentary time, sedentary behaviour patterns and LPA with individual CVD risk factors and functional disability in RA.

\section{Methods}

\section{Participants}

Participants were patients recruited to the Physical Activity in Rheumatoid Arthritis (PARA) study (Trial Number: ISRCTN04121489), a randomised controlled trial with the primary aim of improving cardiorespiratory fitness among RA patients [50]. All participants fulfilled diagnostic criteria for RA as outlined by the American College of Rheumatology [51]. Exclusion criteria to the PARA study were recent joint surgery (in preceding 6 months), fibromyalgia and co-morbidity incompatible with exercise as per the ACSM guidelines. This study utilised baseline data collected during the PARA study. The study was approved by the local NHS Research Ethics Committee.

\section{Recruitment and protocol}

Patients attending Rheumatology outpatient clinics at Russells Hall Hospital (Dudley, England), were provided with study information sheets. Interested participants $(N=97$, mean age $( \pm$ SD $)=58.7 \pm 10.18$ years $)$ provided informed consent and attended two appointments one week apart. During the first visit, participants 
provided a fasted blood sample and were fitted with an accelerometer to wear for the next seven days. Participants attended their second visit one week later to return accelerometers, and undergo assessments to evaluate factors associated with their physical and cardiovascular health (e.g., height and weight [bodymass-index], blood pressure, self-reported functional disability).

\section{Measures \\ Objectively assessed sedentary behaviour patterns, LPA and MVPA}

Sedentary behaviour patterns, LPA and MVPA were assessed using GT3X accelerometers (Actigraph) [52]. The GT3X detects movements over pre-specified time periods called epochs. Movement counts within each epoch are summed and converted to activity counts that are interpreted to determine frequency, intensity and duration of PA and sedentary time engagement. Accelerometers were initialised to measure PA in $60 \mathrm{~s}$ epochs. Participants wore the accelerometer for seven days, on the right hip, removing only for water-based activities (e.g., swimming and bathing).

Accelerometer data reduction Data were downloaded from the GT3X and analysed using the Actilife software (Actilife version 6.2). Time filters were applied to the software, enabling extraction of data pertaining only to waking hours (i.e., 7:00 am - 10:30 pm). Data were cleaned and checked for spurious values and periods of non-wear. Non-wear time was determined by identifying strings of consecutive zeros recorded by the accelerometer for $>60 \mathrm{~min}$, allowing for $2 \mathrm{~min}$ of counts $<100$ [53]. Valid wear criteria was set as $\geq 10 \mathrm{~h}$ wear during waking hours on $\geq 4$ days, including a weekend day [53, 54]. Participants meeting minimum accelerometer wear criteria $(N=61)$, were retained for inclusion in subsequent analyses (excluded, $N=36$ ).

Computation of sedentary behaviour variables, LPA and MVPA Sedentary time, LPA and MVPA were defined as $<100,100-2019$, and $\geq 2020$ counts per minute, respectively [53]. The counts-based method used to quantify time spent in these behaviours is derived from validation studies employing definitions of $\leq 1.5$ (sedentary behaviour), $1.6-2.9$ (LPA) and $\geq 3$ METs (MVPA) [53]. Sedentary breaks were calculated as interruptions in sedentary time (i.e., $\geq 100$ counts per minute) for $\geq 1 \mathrm{~min}$ [41]. Sedentary bout variables were derived by determining the number and length of consecutive zeros recorded by the accelerometer, relative to a $\geq 20 \mathrm{~min}$ bout length [41]. Independent variables computed for statistical analysis were sedentary time $(\mathrm{min} /$ day), the average number of sedentary bouts $\geq 20 \mathrm{~min}$ (number/ day), the average time per sedentary bout $\geq 20 \mathrm{~min}$ (minutes), the number of sedentary breaks (number/day), LPA (min/day) and MVPA (min/day).

\section{0-year risk of CVD}

Global 10-year CVD risk was determined via the calculation of the Q-risk score (Qrisk-2) [55]. Specifically, age, gender, ethnicity, physical assessments (height, weight, systolic blood pressure) total cholesterol/HDL ratio, self-reported smoking status, diabetic status, presence of kidney disease and family history of heart disease were used to determine participants Q-risk-score [55]. Height was measured to the nearest $0.5 \mathrm{~cm}$ using a standard height measure (Seca 214 Road Rod), and weight was determined using digital scales (Tanita Corporation). Blood pressure was assessed using an electronic sphygmomanometer (Datascope Accutor) using procedures previously described [56]. Total cholesterol and high density lipoprotein-cholesterol (HDL-C) were determined from venous blood samples collected in the fasting state ( $\geq 12 \mathrm{~h}$ fast), and analysed using routine laboratory procedures.

\section{Individual CVD risk factors}

Individual CVD risk factors determined from fasted blood samples were; plasma markers of inflammation (C-reactive protein (CRP), erythrocyte sedimentation rate (ESR) and fibrinogen - measured using standard laboratory procedures or ELISAs), plasma lipids (total cholesterol, HDL-C, low density lipoprotein cholesterol (LDL-C) and triglycerides), blood pressure (systolic and diastolic) and plasma glucose and insulin. Homeostasis model assessment (HOMA) was also calculated to determine participants' degree of insulin resistance.

\section{Functional disability}

The Stanford Health Assessment Questionnaire (HAQ) was used to determine participants' degree of functional disability [57]. The HAQ is comprised of 8 sections pertaining to different activities; dressing, arising, eating, walking, hygiene, reach, grip, and activities, Following the stem, "Are you able to....", each section includes 2 or 3 questions (e.g., dressing, 'shampoo your hair'), scored from 0 (without any difficulty) to 3 (unable to do). The score given to each section is the highest score reported within that section. The score is computed as the average of the sum of the scores from all 8 sections.

\section{Statistical analyses}

Data were checked for normality using the KolmogorovSmirnov tests of normality; non-normally distributed data were log transformed (Table 1) and used in subsequent parametric statistical tests. Chi-square tests and one way analyses of variance (ANOVA) were conducted 
Table 1 Descriptive statistics for the total sample and sample stratified by gender

\begin{tabular}{|c|c|c|c|}
\hline & Male $(N=20)$ & Female $(N=41)$ & Total $(N=61)$ \\
\hline Age (years) & $58.85 \pm 9.44$ & $53.00 \pm 13.28$ & $54.92 \pm 12.39$ \\
\hline Height (m) & $1.73 \pm 0.10$ & $1.63 \pm 0.06$ & $1.66 \pm 0.09$ \\
\hline Weight (kg) & $78.13 \pm 10.33$ & $76.81 \pm 19.42$ & $77.23 \pm 16.94$ \\
\hline Ethnicity (\% Caucasian) & 31.1 & 54.1 & 85.2 \\
\hline RA duration (years from diagnosis) & $5.05 \pm 5.44$ & $7.97 \pm 10.34$ & $6.96 \pm 9.01$ \\
\hline \multicolumn{4}{|l|}{ Accelerometer data } \\
\hline Average valid wear time (hours/day) & $12.99 \pm 0.74$ & $13.12 \pm 0.77$ & $13.08 \pm 0.76$ \\
\hline Sedentary time (min/day) & $505.40 \pm 71.45$ & $493.52 \pm 67.24$ & $497.42 \pm 68.28$ \\
\hline a LPA (min/day) & $251.14 \pm 71.79$ & $278.23 \pm 67.22$ & $269.35 \pm 69.35$ \\
\hline${ }^{a}$ MVPA (min/day) & $22.65 \pm 22.53$ & $15.70 \pm 13.77$ & $17.98 \pm 17.26$ \\
\hline \multicolumn{4}{|l|}{ Sedentary behaviour patterns } \\
\hline Number of Sbreaks (average/day) & $79.59 \pm 16.89$ & $86.52 \pm 11.94$ & $84.32 \pm 14.02$ \\
\hline Number of Sbouts $\geq 20$ min (average/day) & $6.37 \pm 2.41$ & $5.45 \pm 1.91$ & $5.75 \pm 2.11$ \\
\hline Average time per Sbouts $\geq 20$ (min) & $31.08 \pm 2.07$ & $30.64 \pm 2.75$ & $30.78 \pm 2.54$ \\
\hline \multicolumn{4}{|l|}{10 year risk of CVD } \\
\hline Q-risk (\% risk of 10 year CVD) & $24.14 \pm 14.82$ & $12.54 \pm 11.05$ & $16.33 \pm 13.45^{* *}$ \\
\hline Self-reported smoking status (\% smokers) & 3.65 & 3.65 & 7.3 \\
\hline Diabetic status (\% diabetic) & 1.8 & 5.5 & 7.3 \\
\hline Presence of kidney disease (\% yes) & 0 & 0 & 0 \\
\hline Family history of heart disease (\% yes) & 118.1 & 36.4 & 54.5 \\
\hline \multicolumn{4}{|l|}{ Individual CVD risk factors } \\
\hline${ }^{\mathrm{a}} \mathrm{CRP}(\mathrm{mg} / \mathrm{L})$ & $6.72 \pm 8.12$ & $7.66 \pm 9.58$ & $7.35 \pm 9.09$ \\
\hline${ }^{\mathrm{a}} \mathrm{ESR}(\mathrm{mm} / \mathrm{h})$ & $10.00 \pm 8.12$ & $16.55 \pm 16.50$ & $14.37 \pm 14.52$ \\
\hline Fibrinogen (g/L) & $4.64 \pm 0.90$ & $4.60 \pm 1.20$ & $4.62 \pm 1.10$ \\
\hline Total cholesterol (mmol/L) & $4.77 \pm 0.70$ & $5.01 \pm 1.09$ & $4.93 \pm 0.98$ \\
\hline${ }^{\mathrm{a}} \mathrm{HDL}-\mathrm{C}(\mathrm{mmol} / \mathrm{L})$ & $1.26 \pm 0.32$ & $1.54 \pm 0.36$ & $1.45 \pm 0.37^{* *}$ \\
\hline${ }^{\mathrm{a}} \mathrm{LDL}-\mathrm{C}(\mathrm{mmol} / \mathrm{L})$ & $2.88 \pm 0.80$ & $2.99 \pm 0.93$ & $2.95 \pm 0.88$ \\
\hline${ }^{a}$ Triglycerides (mmol/L) & $1.39 \pm 0.83$ & $1.05 \pm 0.52$ & $1.16 \pm 0.65$ \\
\hline Syst-BP (mm HG) & $138.07 \pm 19.45$ & $132.32 \pm 16.98$ & $134.08 \pm 17.77$ \\
\hline Diast-BP (mm HG) & $83.13 \pm 9.96$ & $79.18 \pm 8.58$ & $80.39 \pm 9.11$ \\
\hline a Plasma insulin (mmol/L) & $57.35 \pm 26.68$ & $59.32 \pm 55.82$ & $58.67 \pm 47.99$ \\
\hline a Plasma glucose (mmol/L) & $4.65 \pm 0.38$ & $4.80 \pm 1.21$ & $4.75 \pm 1.01$ \\
\hline${ }^{\mathrm{a}}$ HOMA status & $1.70 \pm 0.82$ & $2.11 \pm 3.34$ & $1.97 \pm 2.77$ \\
\hline \multicolumn{4}{|l|}{ Functional Disability } \\
\hline${ }^{\mathrm{a}} \mathrm{HAQ}$ & $1.74 \pm 0.63$ & $1.64 \pm 0.56$ & $1.67 \pm 0.58$ \\
\hline
\end{tabular}

Note: Gender differences indicated as ** $=\mathrm{p}<.01$. Data pertaining to Q-risk-score, ESR, LDL-C, blood pressure and functional disability (HAQ) were available for participants $(N=$ (male, female)) as follows; Q-risk-score, $N=55(18,37), E S R, N=60(20,40)$, LDL-C, $N=60(19,41)$ functional disability, $N=60(20,40)$. Data for Q-risk variables (e.g., smoking status) are reported for participants who have computed Q-risk score $(N=55)$

${ }^{a}$ indicates non-normally distributed data that has been log transformed for inclusion parametric statistical tests. RA rheumatoid arthritis, LPA light physical activity, MVPA moderate-to-vigorous physical activity, Sbreaks sedentary breaks, Sbouts sedentary bouts, CVD cardiovascular disease, Q-risk Q-risk-score, CRP C-reactive protein, ESR erythrocyte sedimentation rate, HDL-C high-density lipoprotein cholesterol, LDL-C low-density lipoprotein cholesterol, Syst-BP systolic blood pressure, Diast-BP diastolic blood pressure, HOMA homeostatic model assessment, HAQ Health Assessment Questionnaire

to determine the occurrence of sample bias related to participant exclusion on the basis of missing accelerometer data. For included participants, descriptive statistics were calculated and one way ANOVAs conducted to determine the presence of gender differences for all measured variables. In addition, one-way ANOVAs were carried out to examine significant differences in targeted outcomes based on current treatment (i.e., Disease Modifying Anti-Rheumatic Drugs (DMARDS), anti-TNF therapy, Non-Steroidal Anti-Inflammatory Drugs (NSAIDs), 
analgesics, cholesterol lowering medication, medication for hypertension). Subsequently, bivariate correlations between targeted variables were computed.

\section{Regression analyses}

Hierarchical linear regressions were carried out to investigate associations between sedentary time, sedentary behaviour patterns and LPA with 10-year CVD risk. Regression models were first adjusted for accelerometerwear time and current treatment as necessary (i.e., where significant differences were observed in Q-risk score based on current treatment) [Model 1]. As age and gender were used to compute participants Q-risk score, regression analyses were not further-adjusted for these variables (i.e., avoiding over-adjustment). Path coefficients $(\beta)$ for sedentary behaviour variables and LPA were examined to determine the strength and direction of the associations with 10-year CVD risk. Significance was set at $p<.05$. The variance in 10 -year CVD risk explained by sedentary time, sedentary bout/break parameters, and LPA engagement were determined via observation of $R^{2}$ values. Where significant associations were observed following initial analyses [i.e., Model 1], models were further adjusted for daily MVPA engagement [Model 2]. Path coefficients were examined to determine whether previously significant associations between sedentary behaviour variables and LPA with 10year CVD risk were maintained after adjustment for MVPA. Path coefficients representing the relationships between MVPA engagement and 10-year CVD risk were also examined to determine the presence of any significant and independent (of sedentary behaviour patterns and LPA) effects of MVPA on 10-year risk of CVD.

Linear regressions were also conducted to examine associations between sedentary time, sedentary behaviour patterns and LPA with 1) individual CVD risk factors and 2) functional disability. Models exploring these relationships were initially adjusted for age, gender, accelerometer wear-time and current treatment (as appropriate) [Model 1], and further adjusted for MVPA where significant associations were observed [Model 2]. Path coefficients were examined to determine the presence of any significant effects of sedentary behaviour patterns and LPA on individual CVD risk factors and functional disability, independent of MVPA (and vice versa). All analyses were conducted using SPSS.

\section{Results}

Included $(N=61)$ versus excluded $(N=36)$ participants did not differ in terms of age, or any of the targeted outcomes (all $p>.05$ ). Chi-square tests confirmed the distribution of males to females was not significantly different among excluded compared to included participants $(X$
$(1)=.27, p=.61)$. No exclusion bias was observed based on ethnicity $(X(1)=.36, p=.55)$.

Descriptive statistics for included participants' data are reported in Table 1. Accelerometer data revealed participants spent approximately 8 to $9 \mathrm{~h} /$ day sedentary (64.84\% of accelerometer wear-time), participated in LPA for $4.5 \mathrm{~h} /$ day (32.22\% of accelerometer wear-time), and broke up their sedentary time approximately 6 times/h. Interpretation of descriptive data indicated $43 \%$ of participants had $\geq 10 \%$ risk of CVD at 10-years based on Qrisk-score. Gender differences were not observed for any sedentary behaviour variables or LPA, but were evident for 10-year risk of $\operatorname{CVD}(F(1,53)=10.62, p=$ $<.01)$ and HDL-C $(F(1,59)=9.91, p=<.01$, Table 1$)$.

At the time of data collection, the number (\%) of participants undertaking different pharmaceutical treatments were as follows; Disease Modifying Anti-Rheumatic Drugs (DMARDS, 53\%), anti-TNF therapy (10\%), Non-Steroidal Anti-Inflammatory Drugs (NSAIDs, 36\%), analgesics (33\%), cholesterol lowering medication (23\%), medication for hypertension (20\%). For each type of medication, one-way ANOVAs demonstrated that overall, targeted outcomes were not significantly different on the basis of current treatment. Exceptions were observed for participants taking cholesterol lowering medication (Q-risk score, $F(1,53)=5.88, p=.02$; $\mathrm{M} \pm \mathrm{SD}$, yes $=23.54 \pm 12.89, \mathrm{no}=13.87 \pm 12.87)$, medication for hypertension (ESR, $F(1,58)=4.49, p=.04 ; \mathrm{M} \pm \mathrm{SD}$, yes $=1.23 \pm 0.33$, no $=0.97 \pm 0.39$; LDL-C, $F(1,58)=4.13, p=$ $<.05 ; \mathrm{M} \pm \mathrm{SD}$, yes $=0.54 \pm 0.11$, no $=0.60 \pm 0.09$; and plasma glucose, $F(1,59)=10.41, p=<.01 ; \mathrm{M} \pm \mathrm{SD}$, yes $=0.73 \pm 0.12$, no $=0.66 \pm 0.04$ ), and anti-TNF therapy (functional disability, $F(1,58)=5.85, p=.02 ; \mathrm{M} \pm \mathrm{SD}$, yes $=1.47 \pm 0.14$, no $=1.25$ \pm 0.22 ). Subsequently, regression analyses which sought to examine associations between sedentary time, sedentary behaviour patterns and LPA with these specific outcomes, were adjusted for current treatment regime as appropriate (see Table 3 legend).

\section{Correlation analyses}

The results of bivariate correlations are displayed in Table 2 . This analysis revealed that average daily sedentary time ( $\mathrm{min} /$ day) and the number of sedentary bouts/ day $\geq 20 \mathrm{~min}$ were significantly positively associated with 10 -year CVD risk. By contrast, daily LPA ( $\mathrm{min} /$ day) was significantly negatively associated with 10-year CVD risk. However, the number of sedentary breaks/day and average sedentary bout length were not related to a 10 -year CVD risk score.

Sedentary time ( $\mathrm{min} /$ day) was not related to any individual CVD risk factors. However, the number of sedentary bouts/day $\geq 20 \mathrm{~min}$ was significantly positively associated with fibrinogen. In addition, LPA ( $\mathrm{min} /$ day) was significantly negatively linked to fibrinogen, and both LPA (min/day) and the number of sedentary 


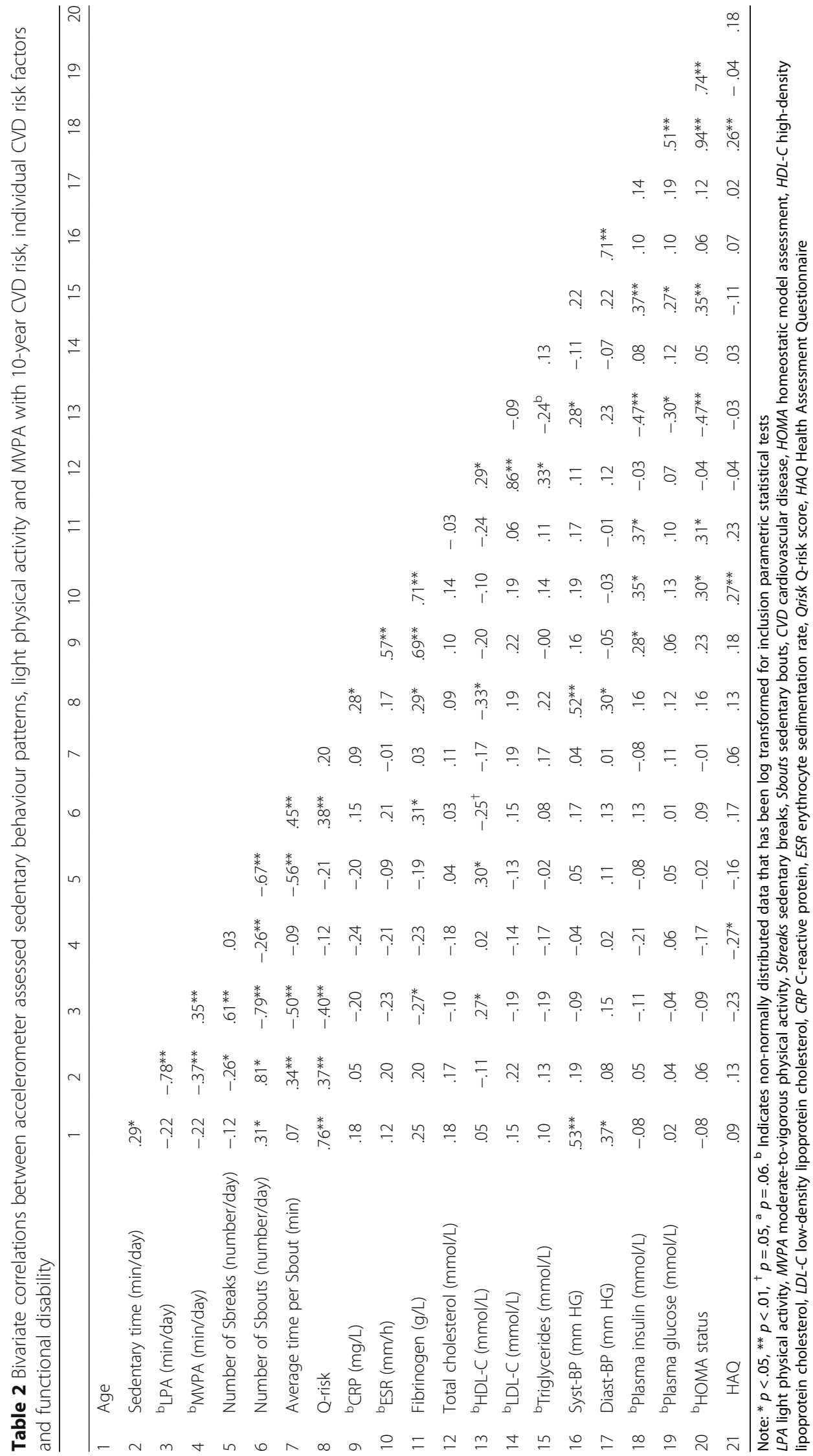


breaks/day demonstrated significant positive associations with HDL-C. Average sedentary bout length was not related to any individual CVD risk factors. Daily MVPA (min/day) was significantly and negatively linked to plasma levels of CRP, ESR and fibrinogen.

No significant associations were observed between any accelerometer derived variables with functional disability. However, functional disability was positively correlated with plasma insulin and ESR.

\section{Regression analysis \\ Model 1}

Results from the regression analyses are reported in Table 3. 10-year CVD risk; the significant positive associations between both daily sedentary time ( $\mathrm{min} /$ day) and the number of sedentary bouts/day $\geq 20$ min with10-year CVD risk were sustained after adjusting for accelerometer wear-time (sedentary time, $\mathrm{R}^{2}=.17$, number of sedentary bouts $\geq 20 \mathrm{~min}, \mathrm{R}^{2}=.15$ ). The significant negative association observed between LPA ( $\mathrm{min} /$ day) and 10year CVD risk was also maintained in wear-time adjusted models $\left(\mathrm{R}^{2}=.18\right)$. Finally, the number of sedentary breaks/day, and the average time per sedentary bout $\geq 20$ min remained unrelated to 10 -year CVD risk in wear-time adjusted models.

Individual CVD risk factors Analyses revealed the previously significant positive associations between both LPA and sedentary breaks with HDL-C (as per bivariate correlations), were attenuated and no longer significant in regression models adjusted for age, gender, accelerometer wear-time and current treatment. However, the number of sedentary bouts/day $\geq 20 \mathrm{~min}$ demonstrated significant positive associations with fibrinogen and ESR in the adjusted models (fibrinogen, $\mathrm{R}^{2}=.06$; ESR, $\mathrm{R}^{2}$ $=.07)$. All other associations between sedentary time (min/day), sedentary bout/break parameters and LPA (min/day) with individual CVD risk factors remained non-significant in age, gender, wear-time and treatment adjusted models.

Functional disability The non-significant associations between sedentary behaviour patterns and LPA engagement with functional disability as observed correlation analyses persisted in age, gender and wear-time and treatment adjusted models.

\section{Model 2 - Adjustment for MVPA}

The significant positive relationships between daily sedentary time ( $\mathrm{min} /$ day) and the number of sedentary bouts/day $\geq 20$ min with 10-year CVD risk, were maintained following adjustment for MVPA (i.e., these associations were independent of levels of MVPA participation, model $R^{2 \Delta}$ MVPA, sedentary time $=0.00$, sedentary bouts $\geq 20 \mathrm{~min}=.00)$. Similarly, the significant negative association between LPA (min/day) and 10-year CVD risk remained after the inclusion of MVPA in regression models $\left(\mathrm{R}^{2 \Delta}\right.$ MVPA $\left.=.00\right)$. The significant positive association between the number of sedentary bouts/ day $\geq 20$ min with fibrinogen and ESR were attenuated and no longer significant following adjustment for MVPA (fibrinogen, $\mathrm{R}^{2 \Delta} \mathrm{MVPA}=.03$; ESR, $\mathrm{R}^{2 \Delta} \mathrm{MVPA}$ $=.02$ ).

No significant associations were observed between daily MVPA with 10-year CVD risk, individual CVD risk factors and functional disability (i.e., where regression models were adjusted for age, gender, accelerometer wear-time, and sedentary behaviour or LPA).

\section{Discussion}

This study is the first to examine the associations between objectively assessed daily sedentary time $(\leq 1.5$ METS), sedentary behaviour patterns, and LPA engagement (1.6 - 2.9 METS) with long-term (10-year) CVD risk in RA. Results revealed daily sedentary time and the number of sedentary bouts/day $\geq 20$ min were positively associated with 10-year CVD risk, with the reverse relationship evidenced for daily LPA participation. Importantly, these significant relationships were observed to be independent of levels of daily MVPA engagement.

Our results suggest that daily sedentary time and LPA engagement may hold implications for 10-year risk of CVD in RA. These findings are aligned with those reported in population based studies, which demonstrate the role of sedentary behaviour and LPA for long-term risk of CVD among adults (e.g., risk of a first atherosclerotic cardiovascular disease event) $[58,59]$. To illustrate the clinical significance of current findings, interpretation of path coefficients indicate that reducing sedentary time by $68 \mathrm{~min} /$ day (i.e., the standard deviation), would equate to a $5.5 \%$ reduction in 10-year CVD risk, regardless of an individual's level of MVPA engagement. Similarly, increasing LPA by the same amount would correspond to a $6 \%$ reduction in 10-year risk of CVD. Of relevance to these findings is the high correlation between LPA and sedentary time that is also observed in this study. This is indicative of a 'displacement association' whereby the action of reducing sedentary time is likely to be synonymous with increasing LPA among people with RA [60]. As such, any improvements in long-term CVD risk derived from increasing participation in LPA, are likely to be equivalent to those resulting from associated reductions in sedentary time among this patient group. Taken together, our results provide the first evidence to suggest that reducing sedentary time engagement and increasing participation in LPA (i.e., "sit less, move more"), may help to attenuate longterm CVD risk in RA. 


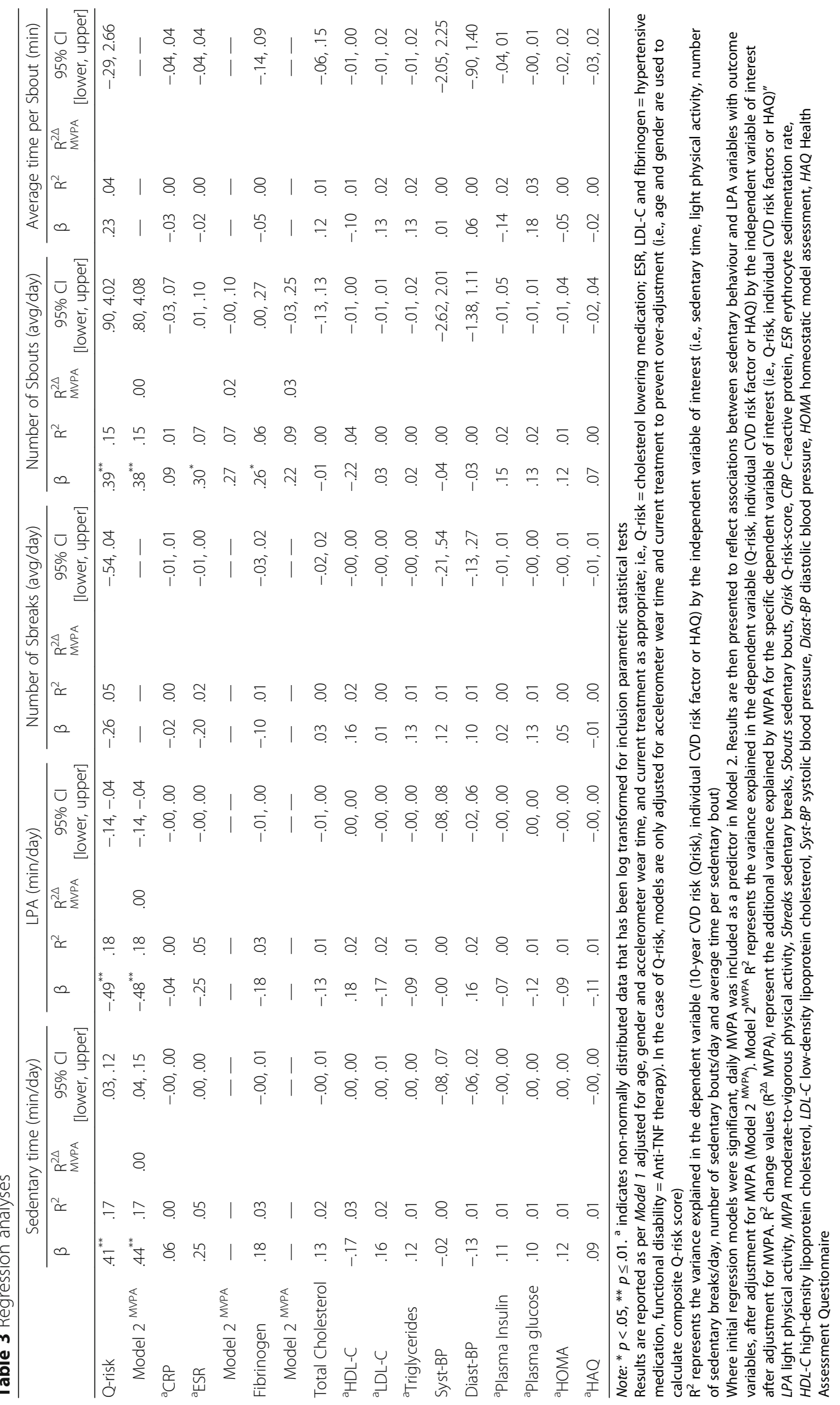


The point that current associations between sedentary time and LPA with 10-year risk of CVD, were observed independently of levels of MVPA participation is particularly pertinent to this contention. Indeed, due to the pain symptomatic of RA and concomitant restricted mobility, people with RA represent a patient group for whom engagement MVPA may be challenging. Consequently, it is likely that for these individuals, reducing sedentary time and increasing engagement in LPA, will be perceived as more feasible than realising higher levels of MVPA participation.

We show for the first time that the number of sedentary bouts/day $\geq 20 \mathrm{~min}$ is positively associated with 10 year CVD risk among RA patients. As such, restricting sedentary bouts to less than $20 \mathrm{~min}$ via interrupting sedentary time with LPA, may be of benefit towards reducing long-term CVD risk for this patient group. This may also represent a palatable health promotion message for people with RA, e.g., aim to break up your sedentary time every $20 \mathrm{~min}$ with LPA. Still, emphasis should also be placed on the duration of the interruption in sedentary time for this population, not just the act of interrupting itself (i.e., a sedentary break). Indeed, no association was observed between the number of sedentary breaks/day and 10-year risk of CVD in this study. Thus, the simple act of interrupting sedentary time may not be sufficient to induce the necessary physiological mechanisms (e.g., improvements in lipid profile) in order to improve long-term CVD risk for RA patients. Further research is therefore warranted to infer the optimal 'sedentary break' duration and associated subsequent intensity of PA engagement likely to induce the required physiological signal to contribute towards a more favourable long-term CVD-risk profile in RA. For example, a recent study revealed that interrupting sedentary time with 2 min periods of light intensity activity was associated with lower systolic blood pressure in overweight and obese adults [61].

Considering that calculated cardiovascular risk score is a composite score, this raises the question of specifically which modifiable factors may contribute towards the significant relationships observed herein. Secondary aims of this study were to explore associations between sedentary behaviour patterns and LPA with individual CVD risk factors, thus enabling the contribution of specific factors to be examined. Our results revealed that both daily sedentary time and LPA demonstrated small to moderate non-significant relationships with total and HDL-cholesterol in adjusted analyses ( $\beta=-.13$ to .18). Whilst not significant, the size of these associations is comparable to significant associations observed in a previous study of RA, in which associations between accelerometer assessed sedentary behaviour and LPA with individual CVD risk factors were examined [62]. Future research should therefore aim to explore the possibility that variability in cholesterol levels as related to levels of sedentary behaviour and LPA, may represent a physiological pathway through which these behaviours are associated with long-term risk of CVD. This proposition is aligned with the hypothesis that down-regulation of lipoprotein lipase (LPL) activity - an enzyme which catalyses the hydrolysis of circulating triglycerides - is a key physiological mechanism underlying the adverse association between sedentary behaviour and CVD risk. Specifically, LPL activity decreases in response to sedentary behaviour, potentially holding adverse implications for cholesterol profile (e.g., lower HDL-cholesterol) [63].

With regards to examination of the relationships between sedentary time, sedentary behaviour patterns and LPA with 1) individual CVD risk factors and 2) functional disability, the lack of significant associations reported herein are inconsistent with findings from extant research [22-24, 36-40, 43, 46, 47, 64]. Only the number of sedentary bouts/day $\geq 20 \mathrm{~min}$ was significantly related to secondary outcomes (i.e., positive associations with fibrinogen and ESR). Previous studies conducted among non-RA populations, have revealed sedentary time, sedentary behaviour patterns, and LPA to be linked to serological markers of CVD and cardiometabolic health (e.g., CRP, HDL-cholesterol, blood pressure and plasma glucose, HOMA status), as well as physical function in both healthy adults and clinical cohorts. Our findings also contradict results reported by Khoja et al., (2016) - the only existing study to examine associations between objectively assessed sedentary behaviour and LPA with markers of CVD and physical function in RA specifically [62]. In the case of Khoja and colleagues (2016), incongruent findings may represent inconsistencies with regards to the way sedentary behaviour and LPA were defined. In this study, we applied the widely accepted $\leq 1.5$ MET definition of sedentary behaviour, as advocated by the sedentary behaviour research network [35], and considered LPA to represent activities requiring $1.6-2.9$ METs $[65,66]$. In contrast, Khoja et al., (2016) defined sedentary behaviour and LPA as activities requiring < $1 \mathrm{MET}$, and $1-2.9 \mathrm{METS}$, respectively. As a consequence, this may have resulted in exclusion of common sedentary behaviours requiring energy expenditure of 1 - 1.5 METS (e.g., sitting and reading a book or typing $[65,67])$, and subsequent underestimation of sedentary time engagement/overestimation of LPA.

Still, current results diverge from those observed among such studies of non-RA populations, in which sedentary behaviour ( $\leq 1.5$ METs) and LPA (1.6-2.9 METS) are reported to be related to CVD risk profile and physical function $[22-24,36-40,43,46,47,64]$. The absence of significant relationships in the current study, may therefore be due in part to the disproportionately higher levels 
of inflammation observed in RA, relative to other populations. That is, the effects of sedentary behaviour and LPA on specific serological markers of CVD and physical function, may be comparatively small when considering the chronic and elevated-inflammatory disease-state characteristic of RA. Thus, in order to reduce systemic inflammation, and improve physical function in RA, the dose and intensity of PA engagement may need to be higher than reported for non-RA populations (i.e., MVPA vs LPA, respectively).

Limitations to the current study include a restricted sample size following data reduction procedures, and a cross-sectional study design. As outlined, longitudinal and experimental studies among larger samples are therefore required to confirm the relationships reported herein. Still, this is the first study to employ accelerometers to explore the implications of sedentary behaviour patterning and LPA for cardiovascular health among RA patients. We also acknowledge that conducting repeated regression analyses to examine the hypothesised associations might have inflated the chance of Type 1 error. However, this is the first study to examine relationships between sedentary behaviour patterns and LPA across a broad range of CVD related outcomes in RA. Therefore, we believe that it is of interest to analyse and report results pertaining to all relevant CVD variables examined within the PARA study, even in the instance that null or significant relationships were observed. Finally, the use of the GT3X in this study means the definition of sedentary behaviour employed considers only energy expenditure (i.e., $\leq 1.5 \mathrm{METs}$ ). That is, we were not able to examine whether behaviours characterised by $\leq 1.5$ METS ( $<100$ counts per minute), occurred whilst sitting/lying vs. standing. Still, studies to date examining the implications of sedentary behaviour for health across diverse populations have largely employed accelerometers to measure sedentary time [52]. Thus, the results presented herein facilitate comparisons with other relevant literature. Nevertheless, future studies should seek to employ devices that enable assessment of posture (e.g., the activPAL) alongside traditional accelerometry based approaches used to measure sedentary time, in order to more accurately determine the implications of sedentary behaviour for health outcomes in RA.

\section{Conclusion}

In conclusion, findings suggest that decreasing sedentary time and increasing participation in LPA may contribute towards reduced 10-year risk of CVD amongst patients with RA, independently of levels of MVPA engagement. In particular, limiting sedentary periods to bouts $\leq 20$ min in length, may attenuate 10-year CVD risk for this patient group. Intervention and experimental studies which examine the specific mechanisms responsible for contributing towards a more favourable CVD risk profile are necessary to extend these findings.

\section{Abbreviations \\ ANOVA: Analysis of variance; CRP: C-reactive protein; CVD: Cardiovascular disease; ESR: Erythrocyte sedimentation rate; HAQ: Health assessment Questionnaire; HDL-C: High-density lipoprotein cholesterol; \\ HOMA: Homeostasis models assessment; LDL-C: low-density lipoprotein cholesterol; LPA: Light physical activity; LPL: Lipoprotein Lipase; \\ METS: Metabolic equivalents; MVPA: Moderate-to-vigorous physical activity; PA: Physical activity; RA: Rheumatoid Arthritis}

\section{Acknowledgements}

The authors would like to acknowledge all of the participants who gave their time to participate in the study. In addition, we would like to thank the staff at the Clinical Research Unit and Russells Hall Hospital who provided assistance with data collection.

\section{Funding}

The Physical Activity in Rheumatoid Arthritis study (PARA) was funded by the Medical Research Council. This Trial is registered at http://www.isrctn.com (Trial Number: ISRCTN04121489).

\section{Availability of data and materials}

The datasets generated during and/or analysed during the current study are not publicly available, but are available from the corresponding author on reasonable request.

\section{Author's contributions}

SF, GM and JVVZ led the structuring and writing of the manuscript. GM, PR, $J V V Z, A O$ and SF organised the data collection. GK contributed towards the clinical aspects of the work. SF, JD, GK, GM and JVVZ contributed towards forming the purpose of the study. JD is the principle investigator on the RCT referred to in the manuscript (the PARA study). All authors were involved in the design of the study, were actively involved in manuscript revision

throughout the writing process, and read and approved the final manuscript.

\section{Competing interests}

The authors declare that they have no competing interests.

\section{Consent for publication}

Not applicable

\section{Ethics approval and consent to participate}

Ethical approval was obtained from the South-Birmingham Research Ethics Committee (protocol number 10/H1206/59). All participants provided informed consent to participate.

\section{Publisher's Note}

Springer Nature remains neutral with regard to jurisdictional claims in published maps and institutional affiliations.

\section{Author details \\ ${ }^{1}$ School of Sport, Exercise and Rehabilitation Sciences, University of Birmingham, Birmingham, UK. 'Faculty of Health Education and Wellbeing, University of Wolverhampton, West Midlands, UK. ${ }^{3}$ Department of Rheumatology, Russells Hall Hospital, Dudley Group NHS Foundation Trust, Dudley, UK. ${ }^{4}$ Department for Health, University of Bath, Bath, UK. ${ }^{5}$ Faculty of Biological Sciences, University of Leeds, Leeds, UK.}

Received: 8 September 2016 Accepted: 6 March 2017

Published online: 29 March 2017

\section{References}

1. Machold KP, Nell V, Stamm T, Aletaha D, Smolen JS. Early rheumatoid arthritis. Curr Opin Rheumatol. 2006;18(3):282-8.

2. Lee DM, Weinblatt ME. Rheumatoid arthritis. Lancet. 2001;358(9285):903-11.

3. Uhlig T, Moe RH, Kvien TK. The burden of disease in rheumatoid arthritis. Pharmacoeconomics. 2014;32(9):841-51. 
4. Goodson N, Marks J, Lunt M, Symmons D. Cardiovascular admissions and mortality in an inception cohort of patients with rheumatoid arthritis with onset in the 1980s and 1990s. Ann Rheum Dis. 2005;64(11):1595-601.

5. Kitas GD, Erb N. Tackling ischaemic heart disease in rheumatoid arthritis. Rheumatology (Oxford). 2003;42(5):607-13.

6. Kitas GD, Gabriel SE. Cardiovascular disease in rheumatoid arthritis: state of the art and future perspectives. Ann Rheum Dis. 2010;70(1):8-14.

7. John $\mathrm{H}$, Hale ED, Treharne $\mathrm{GJ}$, et al. Patient evaluation of a novel patient education leaflet about heart disease risk among people with rheumatoid arthritis. Musculoskeletal Care. 2011;9(4):194-9.

8. Kerola AM, Kauppi MJ, Kerola T, Nieminen TVM. How early in the course of rheumatoid arthritis does the excess cardiovascular risk appear? Ann Rheum Dis. 2012;71(10):1606-15.

9. Gonzalez A, Icen M, Kremers HM, et al. Mortality trends in rheumatoid arthritis: the role of rheumatoid factor. J Rheumatol. 2008:35(6):1009-14.

10. Avina-Zubieta JA, Thomas J, Sadatsafavi M, Lehman AJ, Lacaille D. Risk of incident cardiovascular events in patients with rheumatoid arthritis: a metaanalysis of observational studies. Ann Rheum Dis. 2012;71(9):1524-9.

11. Metsios GS, Stavropoulos-Kalinoglou A, Panoulas VF, et al. Association of physical inactivity with increased cardiovascular risk in patients with rheumatoid arthritis. Eur J Cardiovasc Prev Rehabil. 2009:16(2):188-94.

12. Plasqui $\mathrm{G}$. The role of physical activity in rheumatoid arthritis. Physiol Behav. 2008;94(2):270-5.

13. Verhoeven F, Tordi N, Prati C, Demougeot C, Mougin F, Wendling D. Physical activity in patients with rheumatoid arthritis. Joint Bone Spine. 2016;83(3):265-70

14. Turesson C, Matteson EL. Cardiovascular risk factors, fitness and physical activity in rheumatic diseases. Curr Opin Rheumatol. 2007;19(2):190-6.

15. Prioreschi A, Hodkinson B, Tikly M, McVeigh JA. Changes in physical activity measured by accelerometry following initiation of DMARD therapy in rheumatoid arthritis. Rheumatology. 2014;53(5):923-6.

16. Metsios GS, Stavropoulos-Kalinglou A, Kitas GD. The role of exercise in the management of rhematoid arthritis. Expert Rev Clin Immunol. 2015;11(10):1121-30.

17. Metsios GS, Stavropoulos-Kalinoglou A, van Zanten JJCS, et al. Rheumatoid arthritis, cardiovascular disease and physical exercise: a systematic review. Rheumatology. 2008;47(3):239-48.

18. Zuzana DJ, Munneke M, Zwinderman AH, et al. Is a long-term high-intensity exercise program effective and safe in patients with rheumatoid arthritis?: Results of a randomized controlled trial. Arthritis Rheum. 2003;48(9):2415-24.

19. Rahnama N, Mazloum V. Effects of strengthening and aerobic exercises on pain severity and function in patients with knee rheumatoid arthritis. Int J Prev Med. 2012;3(7):493-8.

20. Van Den Ende CH, Vliet Vlieland TP, Munneke M, Hazes JM. Dynamic exercise therapy in rheumatoid arthritis: a systematic review. Rheumatology. 1998;37(6):677-87.

21. Green AN, McGrath R, Martinez V, Taylor K, Paul DR, Vella CA. Associations of objectively measured sedentary behavior, light activity, and markers of cardiometabolic health in young women. Eur J Appl Physiol. 2014;114(5):907-19.

22. Buman MP, Hekler EB, Haskell WL, et al. Objective light-intensity physical activity associations with rated health in older adults. Am J Epidemiol. 2010; 172(10):1155-65.

23. Marques EA, Baptista F, Santos DA, Silva AM, Mota J, Sardinha LB. Risk for losing physical independence in older adults: the role of sedentary time, light, and moderate to vigorous physical activity. Maturitas. 2014;79(1):91-5.

24. Buman MP, Winkler EAH, Kurka JM, et al. Reallocating time to sleep, sedentary behaviors, or active behaviors: associations with cardiovascular disease risk biomarkers, NHANES 2005-2006. Am J Epidemiol. 2014;179(3): 323-34

25. Gando Y, Yamamoto K, Murakami H, et al. Longer time spent in light physical activity is associated with reduced arterial stiffness in older adults. Hypertension. 2010;56(3):540-6.

26. Gando $Y$, Murakami $H$, Kawakami R, et al. Light-intensity physical activity is associated with insulin resistance in elderly Japanese women independent of moderate-to vigorous-intensity physical activity. J Phys Act Health. 2014;11(2):266-71

27. Howard B, Winkler EA, Sethi P, et al. Associations of Low- and high-intensity light activity with cardiometabolic biomarkers. Med Sci Sports Exerc. 2015;47(10):2093-101.

28. Kim J, Tanabe K, Yokoyama N, Zempo H, Kuno S. Objectively measured light-intensity lifestyle activity and sedentary time are independently associated with metabolic syndrome: a cross-sectional study of Japanese adults. Int J Behav Nutr Phys Act. 2013;10(1):1-7.
29. Sokka T, Hakkinen A, Kautiainen $H$, et al. Physical inactivity in patients with rheumatoid arthritis: data from twenty-one countries in a cross-sectional, international study. Arthritis Rheum. 2008;59(1):42-50.

30. Lee J, Dunlop D, Ehrlich-Jones L, et al. Public health impact of risk factors for physical inactivity in adults with rheumatoid arthritis. Arthritis Care Res. 2012;64(4):488-93.

31. Yu CA, Rouse PC, Van Veldhuijzen ZJJ, et al. Subjective and objective levels of physical activity and their association with cardiorespiratory fitness in rheumatoid arthritis patients. Arthritis Res Ther. 2015;17(1):59.

32. Owen N, Sparling PB, Healy GN, Dunstan DW, Matthews CE. Sedentary behavior: emerging evidence for a New health risk. Mayo Clin Proc. 2010; 85(12):1138-41.

33. Haskell WL, Lee IM, Pate RR, et al. Physical activity and public health: updated recommendation for adults from the American College of Sports Medicine and the American Heart Association. Med Sci Sports Exerc. 2007; 39(8):1423-34

34. World Health Organisation. Physical activity and health in Europe: evidence for action. Denmark: WHO Regional Offices for Europe; 2006 http://www.euro.who.int/__data/assets/pdf_file/0011/87545/E89490.pdf. Accessed 6 Jan 2016

35. Sedentary Behaviour Research Network. Letter to the editor: standardized use of the terms 'sedentary' and 'sedentary behaviours'. Appl Physiol Nutr Metab. 2012;37(3):540-2.

36. Lynch BM, Friedenreich CM, Winkler EA, et al. Associations of objectively assessed physical activity and sedentary time with biomarkers of breast cancer risk in postmenopausal women: findings from NHANES (2003-2006). Breast Cancer Res Treat. 2011;130(1):183-94.

37. Bankoski A, Harris TB, McClain JJ, et al. Sedentary activity associated with metabolic syndrome independent of physical activity. Diabetes Care. 2011:34(2):497-503.

38. Biswas A, Oh Pl, Faulkner GE, et al. Sedentary time and its association with risk for disease incidence, mortality, and hospitalization in adults: a systematic review and meta-analysis. Ann of Intern Med. 2015;162(2): 123-32

39. Rosenberg DE, Bellettiere J, Gardiner PA, Villarreal VN, Crist K, Kerr J. Independent associations between sedentary behaviors and mental, cognitive, physical, and functional health among older adults in retirement communities. J Gerontol A Biol Sci Med Sci. 2016;11(1):78-83.

40. Santos DA, Silva AM, Baptista F, et al. Sedentary behavior and physical activity are independently related to functional fitness in older adults. Exp Gerontol. 2012;47(12):908-12

41. Carson V, Wong SL, Winkler E, Healy GN, Colley RC, Tremblay MS. Patterns of sedentary time and cardiometabolic risk among Canadian adults. Prev Med. 2014;65:23-7.

42. Dunstan DW, Kingwell BA, Larson R, et al. Breaking up prolonged sitting reduces postprandial glucose and insulin responses. Diabetes Care. 2012:35(5):976-83.

43. Sardinha LB, Ekelund U, dos Santos L, Cyrino ES, Silva AM, Santos DA. Breaking-up sedentary time is associated with impairment in activities of daily living. Exp Gerontol. 2015;72:57-62.

44. Gennuso KP, Thraen-Borowski KM, Gangnon RE, Colbert LH. Patterns of sedentary behavior and physical function in older adults. Aging Clin Exp Res. 2016:28(5):943-50.

45. Davis MG, Fox KR, Stathi A, Trayers T, Thompson JL, Cooper AR. Objectively measured sedentary time and its association with physical function in older adults. J Aging Health. 2014;22(4):474-81.

46. Healy GN, Matthews CE, Dunstan DW, Winkler EA, Owen N. Sedentary time and cardio-metabolic biomarkers in US adults: NHANES 2003-06. Eur Heart J. 2011;32(5):590-7.

47. Brocklebank LA, Falconer CL, Page AS, Perry R, Cooper AR. Accelerometermeasured sedentary time and cardiometabolic biomarkers: a systematic review. Prev Med. 2015:76:92-102.

48. Sardinha LB, Santos DA, Silva AM, Baptista F, Owen N. Breaking-up sedentary time is associated with physical function in older adults. $J$ Gerontol A Biol Sci Med Sci. 2015;70(1):119-24

49. Fenton SA, Kitas GD. Rheumatoid arthritis: sedentary behaviour in RA - a new research agenda. Nat Rev Rheumatol. 2016;12(12):698-700

50. Rouse PC, van Zanten Veldhuijzen JJCS, Metsios GS, et al. Fostering autonomous motivation, physical activity and cardiorespiratory fitness in rheumatoid arthritis: protocol and rationale for a randomised control trial. BMC Musculoskelet Disord. 2014;15:445. 
51. Arnett FC, Edworthy SM, Bloch DA, et al. The american rheumatism association 1987 revised criteria for the classification of rheumatoid arthritis. Arthritis Rheum. 1988;31:315-24.

52. Atkin AJ, Gorely T, Clemes SA, et al. Methods of measurement in epidemiology: sedentary behaviour. Int J Epidemiol. 2012;41(5):1460-71.

53. Troiano RP, Berrigan D, Dodd KW, Masse LC, Tilert T, Mcdowell M. Physical activity in the United States measured by accelerometer. Med Sci Sports Exerc. 2008;40(1):181-8.

54. Semanik PAME, Song JING, Chang RW, Manheim LARR, Ainsworth BE, Dunlop DORO. Assessing physical activity in persons with rheumatoid arthritis using accelerometry. Med Sci Sports Exerc. 2010;42(8):1493-501.

55. Hippisley-Cox J, Coupland C, Vinogradova Y, et al. Predicting cardiovascular risk in England and Wales: prospective derivation and validation of QRISK2. BMJ. 2008;336(7659):1475-82.

56. Panoulas VF, Douglas KM, Milionis HJ, et al. Prevalence and associations of hypertension and its control in patients with rheumatoid arthritis. Rheumatology (Oxford). 2007:46(9):1477-82.

57. Kirwan JR, Reeback JS. Stanford health assessment questionnaire modified to assess disability in British patients with rheumatoid arthritis. Rheumatology. 1986;25(2):206-9.

58. Loprinzi PD, Davis RE. Daily movement patterns and predicted 10-yr risk for a first atherosclerotic cardiovascular disease (ASCVD) event using pooled cohort risk equations among US adults. Prev Med. 2015;81:78-81.

59. Maddison R, Jiang Y, Foley L, Scragg R, Direito A, Olds T. The association between the activity profile and cardiovascualr risk. J Sci Med Sport. 2016;19(8):605-10

60. Mansoubi M, Pearson N, Biddle SJH, Clemes S. The relationship between sedentary behaviour and physical activity in adults: a systematic review. Prev Med. 2014;69:28-35.

61. Larsen RN, Kingwell BA, Sethi P, Cerin E, Owen N, Dunstan DW. Breaking up prolonged sitting reduces resting blood pressure in overweight/obese adults. Nutr Metab Cardiovasc Dis. 2014;24(9):976-82.

62. Khoja SS, Almeida GJ, Chester Wasko M, Terhorst L, Piva SR. Association of light-intensity physical activity with lower cardiovascular disease risk burden in rheumatoid arthritis. Arthritis Care Res. 2016;68(4):424-31.

63. Hamilton MT, Hamilton DG, Zderic T. Role of low energy expenditure and sitting in obesity, metabolic syndrome and type 2 diabetes and cardiovascular disease. Diabetes. 2007;56(11):2655-67.

64. Henson J, Yates T, Biddle SJH, et al. Associations of objectively measured sedentary behaviour and physical activity with markers of cardiometabolic health. Diabetologia. 2013;56(5):1012-20.

65. Ainsworth BE, Haskell WL, Whitt MC, et al. Compendium of physical activities: an update of activity codes and MET intensities. Med Sci Sports Exerc. 2000;32:5498-504.

66. Ainsworth BE, Haskell WL, Leon AS, et al. Compendium of physical activities: classification of energy costs of human physical activities. Med Sci Sports Exerc. 1993;25(1):71-80.

67. Newton RL, Han $\mathrm{H}$, Zderic T, Hamilton M. The energy expenditure of sedentary behavior: a whole room calorimeter study. PLoS One. 2013;8(5):e63171.

\section{Submit your next manuscript to BioMed Central and we will help you at every step:}

- We accept pre-submission inquiries

- Our selector tool helps you to find the most relevant journal

- We provide round the clock customer support

- Convenient online submission

- Thorough peer review

- Inclusion in PubMed and all major indexing services

- Maximum visibility for your research

Submit your manuscript at www.biomedcentral.com/submit

) Biomed Central 